\title{
Compact tunable dual-band bandpass filter using open-loop resonator loaded by step impedances cells for multimode WLANs
}

\author{
Mohsen Hayati ${ }^{1 a)}$ and Leila Noori ${ }^{2}$ \\ ${ }^{1}$ Electrical Engineering Department, Faculty of Engineering, Razi University \\ Tagh-E-Bostan, Kermanshah-67149, Iran \\ ${ }^{2}$ Islamic Azad University, Gilan-E-Gharb Branch, Gilan-E-Gharb, Iran \\ a)mohsen_hayati@yahoo.com
}

\begin{abstract}
In this paper, a compact microstrip open-loop resonator loaded by step impedances cells, is designed to achieve dual-band bandpass filter with tunable resonance frequencies. By adjusting the lengths and widths of the step impedances and the dimensions of the open loop resonator, the simulation results shows the dual-band feature of two passbands at 2.4 and almost $5.2 \mathrm{GHz}$ (exactly 5.25). The insertion losses are $0.2 \mathrm{~dB}$ and $0.4 \mathrm{~dB}$ at the $2.4 \mathrm{GHz}$ and $5.2 \mathrm{GHz}$ respectively. There is a good agreement between simulation and measurement results.
\end{abstract}

Keywords: dual-band, step impedance cell, compact size

Classification: Microwave and millimeter wave devices, circuits, and systems

\section{References}

[1] C.-H. Tseng and H.-Y. Shao, "A New Dual-Band Microstrip Bandpass Filter Using Net-Type Resonators," IEEE Microw. Wireless Compon. Lett., vol. 20, no. 4, pp. 196-198, 2010.

[2] M. Jiang, L.-M. Chang, and A. Chin, "Design of Dual-Pass band Microstrip Bandpass filter with mulit-spurious suppression," IEEE Microw. Wireless Compon. Lett., vol. 20, no. 4, pp. 199-201, 2010.

[3] X. Y. Zhang, J.-X. Chen, J. Shi, and Q. Xue, "High-selectivity dualband bandpass filter using asymmetric stepped-impedance resonators," Electronics Letters, vol. 45, no. 1, pp. 63-64, 2009.

[4] X. Chen, G. Han, R. Ma, and J. Gao, "Design of Balanced Dual-Band Bandpass Filter with Self-Feedback Structure," ETRI Jornal, vol. 31, no. 4, pp. 475-477, 2009.

[5] P. Mondal and M.K. Mandal, "Design of Dual-Band Bandpass Filters Using Stub-Loaded Open-Loop Resonators," IEEE Trans. Microw. Theory Tech., vol. 56, no. 1, pp. 150-155, 2008.

[6] M. Hayati and L. Noori, "Compact dual band bandpass filter with ultra wide stopband using open loop resonator loaded by T-shape and open stubs," IEICE Electron. Express, vol. 8, no. 14, pp. 1168-1173, 2011. 


\section{Introduction}

The application of dual-band bandpass filter in modern wireless communication is developed recently. High-speed wireless LANs, offering users up to $54 \mathrm{Mbit} / \mathrm{s}$ wireless access service, operate at 2.4 and $5.2 \mathrm{GHz}$ bands. In [1] the net-type resonator is designed to implement a dual-band bandpass filter, this filter has large size and large insertion loss. In [2] systematic design method for microstrip dual band bandpass filter using stepped impedance resonators (SIRs) is proposed. In this structure the harmonics above the second passband are attenuated successfully but it has very large implementation area and large insertion loss. In [3] a high selectivity dual-band bandpass filter is designed using asymmetric stepped-impedance resonators with five transmission zeros near the passband edges and between two passbands. The problems of the filter are large implementation area and small rejection band between two passband. In [4] a balanced dual-band band-pass filter which consists of symmetric SIRs and open-loop resonators by employing a type of self-feedback structure is proposed. Although an extra transmission zero is introduced near the common-mode resonance frequency, but the selectivity is not good and the insertion losses are large. In [5] three different dual-band bandpass filter are designed using four open loop resonators or two open loop resonators that loaded by open stubs. When four open loop resonators are used large implementations area and large insertion losses is obtained. When two open loop resonators are used,the taped line feed structures are added, which inrceased the filter size again. Also when two open loop resonators are used, four transmission zeros are created that lead to selectivity improvement, but there is no good isolation between two passband and above second band as the level of harmonic attenuation above second band and between the two passband aren't enough. In [6] four open loop resonators are used to obtain dual band bandpass response that lead to increase the filter size. In this paper the microstrip filter is designed using two open loop resonators that loaded by stepped impedance cells to achieve dual band bandpass response with tunable resonance frequencies and good performance.

\section{Filter structure and design}

The open loop resonator is shown in Fig. 1 (a). This resonator is loaded by step impedance cell. The equivalent $L C$ circuit of this resonator is shown in Fig. 1(b), where $C$ and $L$ are capacitance and inductance of the bends respectively. The capacitancec $C_{g}$ and $C_{P}$ corresponds to the equivalent circuit of the gap. The capacitance $C_{1}$ and inductance $L_{1}$ are capacitance and inductance of the step impedance cell. The capacitance $C_{2}$ is related to open end of the step impedance cell. The inductances $L_{3}, L_{4}, L_{5}, L_{9}$ are related to another stubs in open loop. The inductance $L_{10}$ is related to shunt stub as shown in Fig. 2 (a) with electrical length $\theta_{7}$. The resonance frequencies of the proposed resonator can be controlled by the following method:

(i) Resonance frequency controll method using electrical length:

The input admittance of the open end (shown by $\theta_{1}$ ) as shown in Fig. 2 (a) 
a

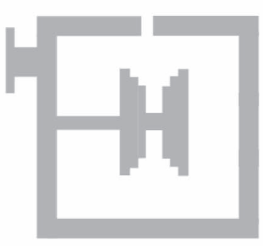

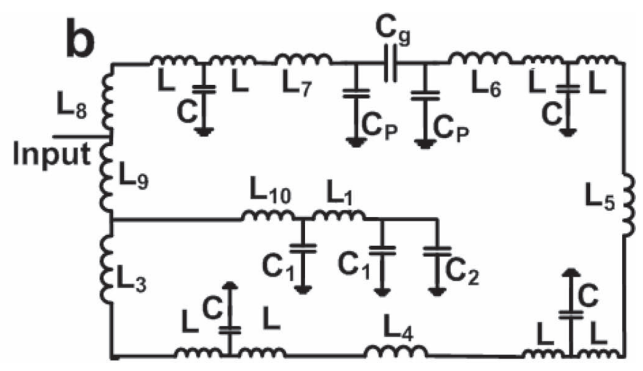

Fig. 1. (a) The open loop resonator loaded by step impedances cell. (b) The equivalent circuit of Fig. 1 (a).

is $[5]$ :

$$
Y_{i n}=J Y \frac{\sum_{i=1}^{20} \tan \left(\theta_{i}\right)}{1-\tan \left(\theta_{1}\right) \sum_{i=2}^{20} \tan \left(\theta_{i}\right)}
$$

Where $Y_{S}=Y_{R}=Y$ and $Y_{R}$ and $Y_{S}$ are the characteristic admittances of the resonator and the stub, respectively. The resonance condition can be obtained from the following:

$$
\sum_{i=1}^{20} \tan \left(\theta_{i}\right)=0
$$

The electrical length $\theta$ is increased when the stub length is increased, so resonance frequency can be controlled by adjusting the physical length.

(ii) Resonance frequency controll metothd using length of parallel paths: The input equivalent admittance viewed from the tapping position to the open ends in the open loop resonator with parallel paths is the admittance of parallel paths [3] that is assumed to be:

$$
Y_{i n, i}=J Y_{i} \cdot \tan \left(\beta . d_{i}\right)
$$

Where $Y_{i}$ and $d_{i}$ are the equivalent characteristic admittance and length of parallel path $h_{i}$ respectively, and $i$ is the number of parallel paths. In the open loop resonator as shown in Fig. 2 (a), three parallel paths exists. When the equivalent characteristic admittance is zero, the resonance condition can be obtained as follow:

$$
Y_{i n, 1}+Y_{i n, 2}+Y_{i n, 3}=0
$$

Therefore the resonance frequency can be controlled by tuning length of parallel paths and the equivalent characteristic admittance of the paths.

(iii) Resonance frequency controll metothd using equivalent circuit: The admittance $y_{t}$ as shown in Fig. $2(\mathrm{~b})$ is the equivalent admittance of shunt stepped impedance cell that is loaded in the open loop. The resonance frequency for this open loop resonator can be controlled by tuning the admittance of shunt stub $y_{t}$ and the admittance of other stubs. For this open loop resonator, the simplified equivalent circuit is shown in Fig. 2 (c). The input equivalent admittance viewed from the tapping position is:

$$
y_{e}=\frac{y_{t} \cdot\left(y_{r}+y_{4}\right)}{y_{t}+y_{r}+y_{4}}
$$




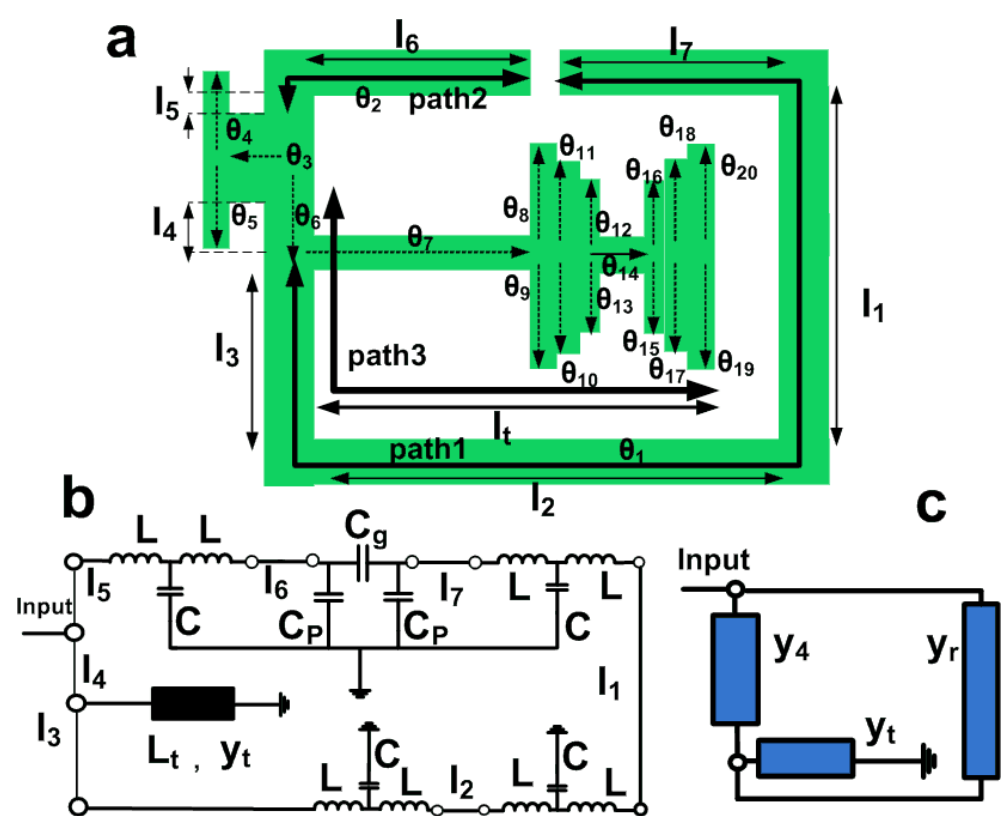

Fig. 2. (a) The open loop resonator that loaded by step impedance cell and tape line structure. (b) The equivalent circuit of Fig. 2 (a). (c) The simpler equivalent circuit.

where $y_{4}$ is the admittance of stub with length $l_{4}$. $y_{t}$ is the admittance of the shunt step impedance that is loaded on the open loop resonator. $y_{r}$ is the total admittance consisting of bents admittance, gap admittance and the admittance of lengths $l_{1}, l_{2}, l_{3}, l_{5}, l_{6}, l_{7} . y_{e}$ is the equivalent admittance viewed from the tapping position. When the equivalent admittance is zero, the resonator operate at resonance frequency. So the resonant conditions are:

$$
y_{r}+y_{4}=0 \quad \text { or } / \text { and } \quad y_{t}=0
$$

The sum $y_{r}+y_{4}$ is the equivalent admittance of the open loop resonator. The admittance of the open loop resonator and shunt step impedance cell are related to the lengths and widths of open loop resonator and shunt step impedance cell. Acording to (i), (ii), and (iii) the resonance frequencies can be controlled by adjusting the admittance of stubs. So the resonance frequency of open loop resonator can be controlled by tuning the lengths and widths of open loop resonator and shunt step impedance cell.

Therefore the dual-band filter structure consist of two open-loop resonators that loaded by step impedance cell are connected together using mixed coupling as shown in Fig. 3 (a) and the dual band bandpass response is obtained as shown in Fig. 3(b). According to the Equations 2 and 4 the resonance frequencies can be controlled by adjusting the parameter $\theta$ and length of path $h_{i}$. The frequency response as function of $l_{1}, l_{2}$ and $l_{3}$ is shown in Fig. 3 (c), also the frequency response as function of $l_{4}$ is shown in Fig. 3 (d). The photograph of fabricated filter is shown in Fig. 3 (e). 

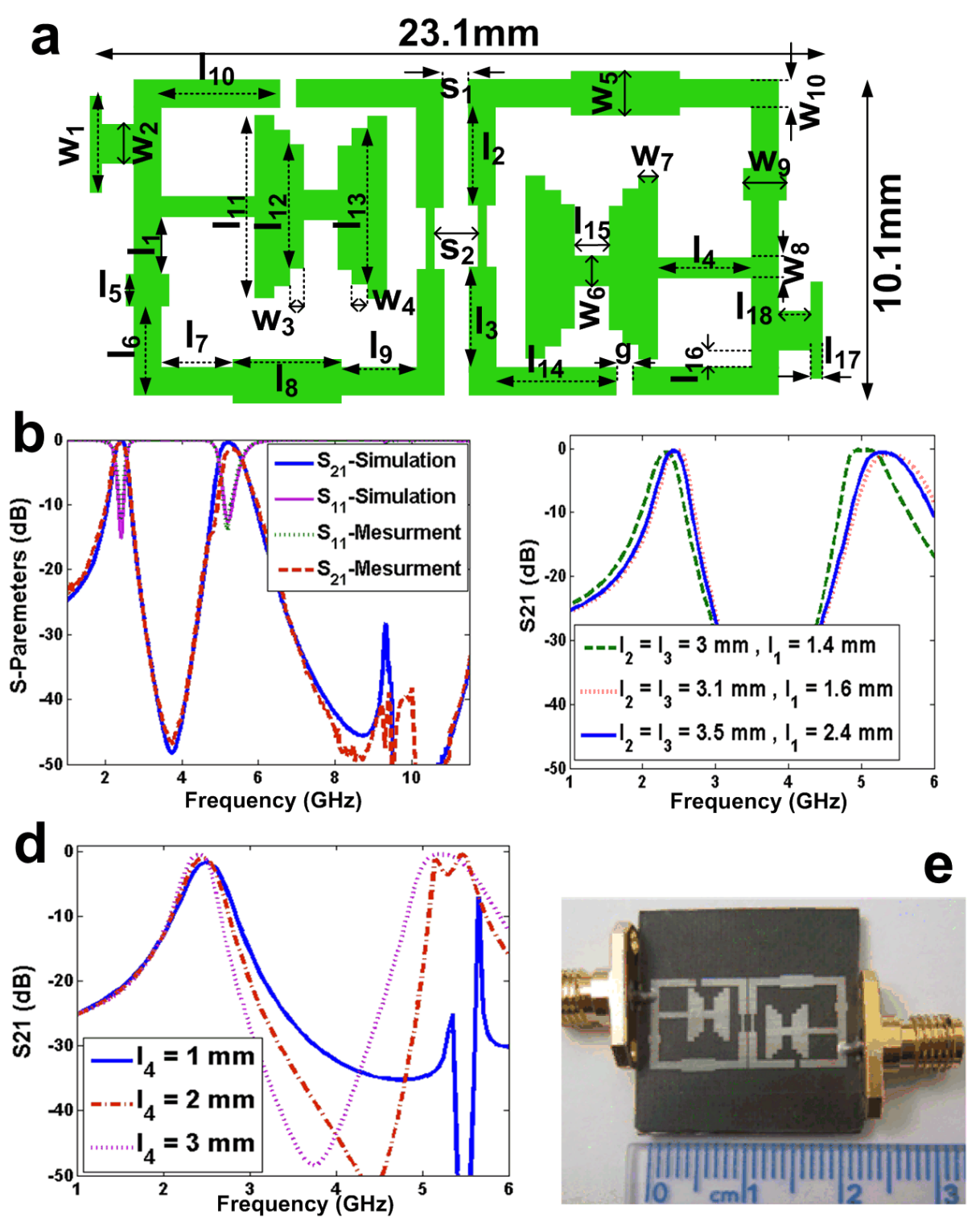

Fig. 3. (a) Two open loop resonator with mixed coupling. (b) The frequency response of two open loop resonator with mixed coupling. (c) The frequency response as the function of $l_{1}, l_{2}$ and $l_{3}$. (d) The frequency response as the function of $l_{4}$. (e) The photograph of fabricated filter.

\section{Simulation and measurement}

The proposed filter is designed and fabricated on a RT Duroid 5880 substrate having dielectric constant $\epsilon_{r}=2.22,15$ mil-thickness and loss tangent of 0.0009. This filter is simulated by using the full wave EM simulator. The dimensions of the proposed structure shown in Fig. 3 (a) are as follow: $l_{1}=$ $1.8 \mathrm{~mm}, l_{2}=3.2 \mathrm{~mm}, l_{3}=3.2 \mathrm{~mm}, l_{4}=3 \mathrm{~mm}, l_{5}=1 \mathrm{~mm}, l_{6}=2.9 \mathrm{~mm}, l_{7}=$ $2.4 \mathrm{~mm}, l_{8}=3.5 \mathrm{~mm} . l_{9}=2.4 \mathrm{~mm}, l_{10}=3.9 \mathrm{~mm}, l_{11}=6 \mathrm{~mm}, L_{12}=4 \mathrm{~mm}$, $l_{13}=5 \mathrm{~mm}, l_{14}=3.9 \mathrm{~mm}, l_{15}=1 \mathrm{~mm}, l_{16}=0.5 \mathrm{~mm}, l_{17}=0.4 \mathrm{~mm}, l_{18}=$ $1 \mathrm{~mm}, w_{1}=3 \mathrm{~mm}, w_{2}=1.3 \mathrm{~mm}, w_{3}=0.5 \mathrm{~mm}, w_{4}=0.5 \mathrm{~mm}, w_{5}=1.4 \mathrm{~mm}$, $w_{6}=1 \mathrm{~mm}, w_{7}=0.7 \mathrm{~mm}, w_{8}=0.7 \mathrm{~mm}, w_{9}=1.3 \mathrm{~mm}, w_{10}=0.9 \mathrm{~mm}, S_{1}=$ 
$0.1 \mathrm{~mm}, S_{2}=0.7 \mathrm{~mm}$ and $\mathrm{g}=0.5 \mathrm{~mm}$. This filter has cut off frequencies at $2.24 \mathrm{GHz}, 2.56 \mathrm{GHz}$ in the first pass band and $4.96 \mathrm{GHz}, 5.55 \mathrm{GHz}$ in the second pass band. Below the first pass band, the minimum attenuation level is $-20 \mathrm{~dB}$ from dc to $1.59 \mathrm{GHz}$. Between the two pass bands, the minimum attenuation level is $-20 \mathrm{~dB}$ from 2.85 to $4.59 \mathrm{GHz}$. Minimum attenuation above the second pass band is $-20 \mathrm{~dB}$ from 6.37 to $11.75 \mathrm{GHz}$. The insertion losses at 2.4 and $5.2 \mathrm{GHz}$ are better than 0.2 and $0.4 \mathrm{~dB}$ respectively, while the return losses in the first and second pass band are better than $-15 \mathrm{~dB}$ and $-12.7 \mathrm{~dB}$ respectively. The filter size is $233.3 \mathrm{~mm}^{2}$. In comparison with the previous works the filter size is smaller and insertion loss is better. Fractional band widths in the first and second band are $13 \%$ and $11 \%$ respectively. This filter has frequency response with a transmission zero between two pass bands, and two transmission zeros above second band. Table I shows the comparison between the proposed filter and previous works.

Table I. Comparison between the proposed filter and previous works.

\begin{tabular}{lllll}
\hline References & $\begin{array}{l}\text { Approximated } \\
\text { size }\left(\mathrm{mm}^{2}\right)\end{array}$ & $\begin{array}{l}\text { First band } \\
\text { insertion } \\
\text { loss }(\mathrm{db})\end{array}$ & $\begin{array}{l}\text { Second band } \\
\text { insertion loss } \\
(\mathrm{db})\end{array}$ & $\begin{array}{l}\text {-20 dB harmonic } \\
\text { attenuation above } \\
\text { second band (Skirt } \\
\text { characteristics) }\end{array}$ \\
\hline $\begin{array}{l}\text { proposed } \\
\text { filter }\end{array}$ & 233.3 & 0.2 & 0.4 & upto $11.75 \mathrm{GHz}$ \\
{$[1]$} & 1327 & 2.65 & 2.44 & upto $6 \mathrm{GHz}$ \\
{$[2]$} & 1794 & 2.12 & 2.33 & upto $20 \mathrm{GHz}$ \\
{$[3]$} & 1277 & 1.1 & 1 & upto $3.5 \mathrm{GHz}$ \\
{$[4]$} & 311 & 1.85 & 1.9 & upto $7 \mathrm{GHz}$ \\
{$[5]$} & 649 & 3.5 & 3.55 & upto $10 \mathrm{GHz}$ \\
{$[6]$} & 496 & 0.2 & 0.9 & upto $20 \mathrm{GHz}$ \\
\hline
\end{tabular}

\section{Conclusion}

A novel dual-band bandpass filter with low insertion losses and compact size is designed and fabricated for high-speed multimode wireless LANs, operated at 2.4 and $5.2 \mathrm{GHz}$ bands. The filter size is $233.3 \mathrm{~mm}^{2}$. It is seen that the designed filter not only have smaller size and good insertion loss but also have good performance in terms of return loss, fractional band width and the harmonics above the second passband are attenuated from 6.37 to $11.75 \mathrm{GHz}$ with minimum attenuation of $-20 \mathrm{~dB}$. 